\title{
Innovative Energy Arbitrage Models and Algorithms for Battery Energy Storage Systems in Electricity Market
}

\author{
Ang Li; Jiming Peng; Lei Fan, Senior Member, IEEE; Pengwei Du, Senior Member, IEEE
}

\begin{abstract}
Maintaining the balance between electricity production and consumption is an essential task in the operations of modern power grids. In recent years, battery energy storage system (BESS) has been gaining more and more attention owning to its decreasing capital cost, high flexibility and short response time. However, there exist several technical challenges in developing accurate models and effective algorithms for the operations of BESS. One major challenge is that in order to precisely describe the changing dynamics of battery status, usually highly non-linear functions and integer variables must be used in the model, leading to optimization models with nonlinear and non-convex objective function/constraints that are difficult to handle.

To address the above challenges, in this paper we first explore the physical law in the battery charging and discharging process to develop a new model which naturally addresses both the charging and discharging processes through a single current decision variable. Then we propose to approximate the dependency relationship between the open circuit voltage (OCV) and the state of charge (SOC) by some linear functions. This leads to a new non-convex quadratic programming model with linear and bilinear constraints (BLCQP) for the identification of the optimal operational strategy for the BESS. To cope with the bilinear constraints in the BLCQP, we introduce a novel transformation technique to transfer the original BLCQP into another equivalent exponential optimization problem with linear constraints (LCEO). A new sequential linear and quadratic programming approach (SLQP) for the LCEO is developed and its convergence is established. Preliminary experiments are conducted to demonstrate the efficacy of the new model and the efficiency of the new algorithm.
\end{abstract}

Index Terms-Battery Energy Storage, Electricity Market, Energy Arbitrage Model, Nonconvex Quadratic Programming.

\section{NOMENCLATURE}

A. Parameters

$\mathcal{T} \quad$ The set of operation time span.

$E C \quad$ The energy capacity of the battery energy storage.

$R \quad$ The electrical resistance of the battery energy storage.

$P_{t}^{c} \quad$ The maximum charging rate for the battery energy storage.

$P_{t}^{d} \quad$ The maximum discharging rate for the battery energy storage.

Ang Li and Jiming Peng is with the Department of Industrial Engineering, University of Houston, Houston, TX 77204. E-mail: ali21@ central.uh.edu, jopeng@central.uh.edu,

Lei Fan (Corresponding Author) is with the Department of Engineering Technology, University of Houston, Houston, TX 77204. Email: 1fan8@central.uh.edu.

Pengwei Du is with Electric Reliability Council of Texas, Austin, TX. Email: Pengwei.Du@ercot.com
$I^{c} \quad$ The maximum charging current for the battery

$I^{d} \quad$ energy storage.

$I^{d} \quad$ The maximum discharging current for the battery

energy storage.

$\lambda_{t} \quad$ Electricity price at period $t$.

$\eta^{c} \quad$ The charging efficiency.

$\eta^{d} \quad$ The discharging efficiency.

$\Delta \quad$ The length of time interval.

B. Decision Variables

$p_{t}^{c} \quad$ The charging power of the battery energy storage at period $t$.

$p_{t}^{d} \quad$ The discharging power of the battery energy storage at period $t$.

$s_{t} \quad$ The state of charge (SOC) of the battery energy storage at time period $t$.

$i_{t} \quad$ The charging/discharging current of the battery energy storage at time period $t$.

$v_{t}^{o c} \quad$ The open circuit voltage of the battery energy storage at time period $t$.

C. Abbreviations

BESS Battery energy storage systems.

OCV The open circuit voltage.

SOC The state of charge.

OCVDF The dependency function of the open circuit voltage on the state of charge.

PAM The power arbitrage model.

VIAM The voltage (V) current (I) arbitrage model.

BLCQP Bi-linear constrained quadratic programming.

LCEO Linear constrained optimization with exponential objective function.

SLQP The sequential linear and quadratic programming algorithm.

\section{INTRODUCTION}

In recent years, due to climate change, extreme weather events have occurred frequently. For example, the 2021 North American winter storm caused widespread blackouts in Texas, which led to heavy casualties and property losses. To slow the process of climate warming, the U.S. government has set a goal of achieving net-zero carbon emissions by 2050. This is a task that cannot be done without the help of renewable energy resources. However, renewable energy resources such as wind energy and solar energy are changing the operational mode of the power system because traditional decision methods and approaches cannot deal with the high volatility and uncertainty of these renewable energy resources [1]. 
In order to maintain the reliability and flexibility of the power system under the high penetration rate of renewable energy, highly flexible resources such as energy storage have become one of the important resources for the system to respond to and track the dynamic changes caused by wind and solar energy [2]. Among different types of energy storage resources such as pump energy storage and compressed air energy storage, battery energy storage has become a popular choice due to its wide range of advantages. For example, unlike pump energy storage plants and compressed air energy storage, which are restricted by geographic locations, battery energy storage can be installed not only in power generation systems, but also in power distribution systems. In addition, the cost of battery energy storage has dropped significantly according to [3].

Battery energy storage resources have been broadly studied in many areas including power system planning [4], power system operations [5], power distribution system [6], bidding strategy [7], virtual power plants [8], and micro-grid systems [9]. In these applications, most researchers use a high level optimization model to describe the battery energy storage operational process, in which the charging and discharging processes are controlled by two different variables [10]. Because the charging and discharging can not happen at the same time, some complementarity conditions are imposed to ensure that at most one of these two variables can take nonzero values. From a mathematical perspective, the complementary conditions can also be modeled using bilinear constraints [11] or binary variables [12]. The presence of massive bilinear constraints has become a major challenge in the development of effective algorithms for these models. Researchers [13] investigated the possibility to get rid of the bilinear constraints and binary variables to have a convex optimization model. However, as pointed by [10], these convex relaxation model will result in impractical solutions which might be infeasible.

Recently, researchers [14] and [15] studied more detailed battery energy storage model with considering the dependency relationship between the open circuit voltage (OCV) and SOC. The open-circuit voltage $v_{o c}$, representing the voltage difference between the electrodes at the open circuit, depends on the SOC. In the remaining parts of this paper, for convenience, we assume the dependency relationship is defined by the so-called open-circuit voltage dependency function (OCVDF). Various OCVDF formulations have been proposed in the literature and most of these formulations are highly non-linear and nonconvex [14], [16]-[20], which further leads to non-convex optimization models with non-convex constraints for the BESS that are difficult to solve. This forms the second major computational challenge in the BESS optimization models. Although [14] and [15] provide a new detailed battery energy storage model, the authors ignored the complementary conditions and did not discuss how to cope with the nonlinearity and nonconvexity arisen from the OCVDF.

The main goal of this work is to address the abovementioned major challenges in the BESS optimization models. To address the first major challenge, we will review the basic BESS optimization model widely used in the literature using two charging/discharging power variables. By exploring the physical principles in the process of battery charging and discharging, we propose to use only a single current variable $\left(i_{t}\right)$ to model both the charging and discharging processes in the BESS. Specifically, $i_{t}>0$ indicates that the battery is in the charging process and $i_{t}<0$ indicates that the battery is in discharging process. Consequentially, such a formulation ensures that both charging and discharging won't happen simultaneously, which provides a natural way to model the charging/discharging process of the battery. Mathematically speaking, this also allows us to get rid of the complimentary conditions (i.e., $p_{t}^{c} p_{t}^{d}=0$ ) in most existing optimization models in the literature and thus help to substantially simplify these models.

To address the second challenge, we observe that one major nonlinearity and non-convexity stem from the OCVDF when the detailed BESS optimization model is applied [19]. However, as pointed out by [21], normally the state of charge is operated in the range $[20 \%, 80 \%]$ because the lifetime of the battery will be reduced significantly when it is operated outside such a range. When the SOC is restricted to the range of $[20 \%, 80 \%]$, we can use some linear functions to obtain a very good approximation to the original nonlinear OCVDF $(g(s))$. This can be verified from our experiment. For example, if we use the OCVDF defined in [22] as the original OCVDF and apply the least squares method in Banach space [23] to obtain the linear approximate function, then we need to solve the following optimization problem to estimate the two parameters $c_{0}$ and $c_{1}$ in the approximate linear function.

$$
\begin{aligned}
\min _{s_{u}, \bar{g}} & L(\bar{g})=\int_{0.2}^{0.8}(\bar{g}(s)-g(s))^{2} d s . \\
\text { s.t. } & \bar{g}(s)=c_{0}+c_{1} s .
\end{aligned}
$$

By solving the above convex optimization problem, we obtain the approximation function $\bar{g}(s)=14.8629 s+126.196$. The following figure shows the original OCVDF $g(s)$ and its linear approximation $\bar{g}(s)$.

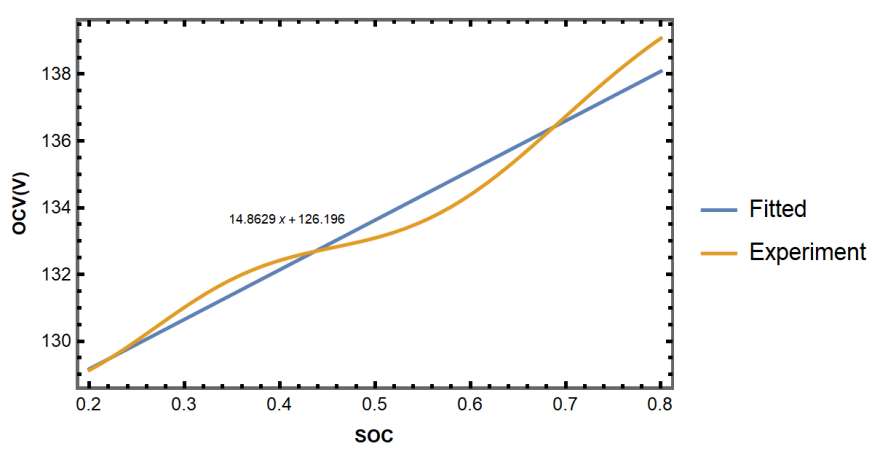

Fig. 1: The OCVDF and its linear approximation

To estimate how the linear function approximates the OCVDF, we compute the maximal relative error via solving the following optimization problem:

$$
\rho=\max _{s \in[0.2,0.8]} \frac{|\bar{g}(s)-g(s)|}{g(s)} .
$$


In the experiment, we obtain $\rho=0.597462 \%$. It follows

$$
0.994 \leq 1-\rho \leq \frac{\bar{g}(s)}{g(s)} \leq 1+\rho \leq 1.006, \quad \forall s \in[0.2,0.8] \text {. }
$$

The above relation demonstrates that though the linear approximation may lose some accuracy at certain points, it does provide a very good approximation to the original OCVDF as a whole.

As we shall see in our later analysis, even when the OCVDF is linear, the resulting BESS optimization model reduces to a non-convex quadratic programming with linear and bilinear constraints (BLCQP), which is still hard to solve. The bilinear term results from the product of the voltage and current. To cope with the bilinear constraints in the corresponding BESS optimization model, we will introduce a novel nonlinear transformation to reformulate the original BLCQP into another equivalent exponential optimization problem with only linear constraints (LCEO). We then modify the standard sequential quadratic programming approach to develop the so-called sequential linear/quadratic programming (SLQP) for the reformulated LCEO model and establish the convergence for the proposed SLQP. We also conduct numerical experiments to verify the efficacy of our new BESS optimization model and the efficiency of the new SLQP algorithm.

This paper is organized as follows. In Section II, we first describe the basic BESS optimization model in the literature and then explore the physical laws in the charging/discharging process to simplify the basic model. In Section III, we introduce a nonlinear transformation technique to reformulate the original BLCQP as the equivalent LCEO. In Section IV, we describe the SLQP approach for the reformulated LCEO model and establish the convergence of the proposed algorithm. In Section V, we present a case study using electricity price and battery data to demonstrate the efficacy of our new models and techniques. In section VI, we conclude the paper by summarizing the topics discussed.

\section{BAtTery ARbitrage Optimization Model in ELECTRICITY MARKET}

In the literature [10], [14], [16], the essential part of BESS power arbitrage model (PAM) can be extracted as follows:

$$
\begin{gathered}
(P A M) \min _{p_{t}^{d}, p_{t}^{c}, s_{t}} \sum_{t \in \mathcal{T}} \lambda_{t}\left(p_{t}^{c}-p_{t}^{d}\right) \\
\text { s.t. } \quad p_{t}^{c} p_{t}^{d}=0, \forall t \in \mathcal{T} ; \\
\quad 0 \leq p^{c} \leq P_{t}^{c}, \forall t \in \mathcal{T} ; \\
0 \leq p^{d} \leq P_{t}^{d}, \forall t \in \mathcal{T} ; \\
s^{l} \leq s_{t} \leq s^{u}, \forall t \in \mathcal{T} ; \\
E C s_{t+1}=E C s_{t}+p_{t}^{c} \eta^{c} \Delta-\frac{p_{t}^{d}}{\eta^{d}} \Delta, \forall t \in \mathcal{T} .
\end{gathered}
$$

In the electricity market, the BESS owners use the PAM to help them make decisions. In this PAM, the objective function is to minimize the costs (or maximize the profits equivalently) of the BESS in the electricity market. This PAM includes the complementary constraints (1b), the charging/discharging rates limits (1c-1d), the operational ranges constraints (1e) and the state of charge balance (1f). This PAM has been widely used in different power system applications such as unit commitment, economic dispatch, transmission planning, etc. However, this model ignores the relationship between the state of charge and voltage of the storage and thus significantly simplifies the battery energy storage physical operation process. As pointed out by Arroyo et al. in [10], this simplified model may lead to infeasible dispatch strategy for the field operations of the BESS.

\section{A. Physical Model}

In this section, we propose a brand new BESS schedule model based on equivalent circuit model by using the fundamental physical laws. In the following, we always assume the time span we study is $\mathcal{T}=\{1,2,3, \ldots, T\}$.

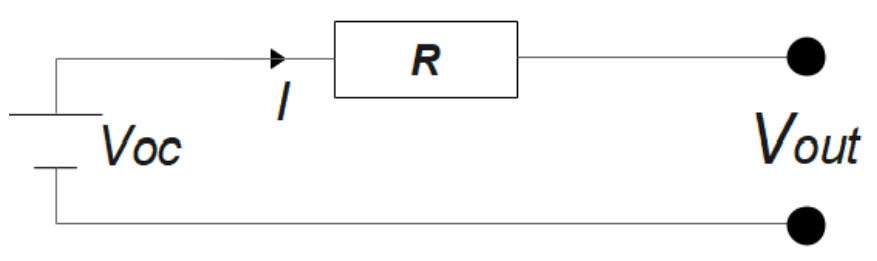

Fig. 2: Equivalent Circuit

Figure 2 shows the circuit of BESS. Let $R$ be the equivalent resistance. When BESS is in the discharging status, we have the following equations.

$$
\begin{aligned}
& v_{t}^{o c, d}=v_{t}^{\text {out }, d}+i_{t}^{d} R ; \\
& p_{t}^{d}=v_{t}^{\text {out }, d} i_{t}^{d} .
\end{aligned}
$$

When BESS is in the charging status, we have the following equations.

$$
\begin{aligned}
& v_{t}^{o c, c}=v_{t}^{o u t, c}-i_{t}^{c} R ; \\
& p_{t}^{c}=v_{t}^{o u t, c} i_{t}^{c} .
\end{aligned}
$$

Now, we use general formulation to characterize both the charging and discharging status. The positive value of current $i$ represents that the battery is charging. Then negative value of current $i$ represents that the battery is discharging. Then we have.

$$
\begin{aligned}
& v_{t}^{\text {oc }}=v_{t}^{\text {out }}-i_{t} R ; \\
& p_{t}^{\text {out }}=v_{t}^{\text {out }} i_{t} .
\end{aligned}
$$

Using this general formulation, we may express the objective function (1a) in PAM as $\sum_{t \in \mathcal{T}} \lambda_{t} p_{t}^{o u t}=\sum_{t \in \mathcal{T}} \lambda_{t}\left(v_{t}^{o c} i_{t}+\right.$ $i_{t}^{2} R$ ), and eliminate the complimentary constraint (1b). By using the equivalent circuit model (2a) - (2b), we can reformulate the PAM as the voltage (V) current (I) arbitrage model (VIAM) as follows.

$$
\begin{aligned}
(V I A M) & \min _{v_{t}^{o c}, i_{t}, s_{t}} \sum_{t \in \mathcal{T}} \lambda_{t}\left(v_{t}^{o c} i_{t}+i_{t}^{2} R\right) \\
\text { s.t. } \quad & E C s_{t+1}=E C s_{t}+v_{t}^{o c} i_{t} \Delta, \forall t \in \mathcal{T} ; \\
& v_{t}^{o c}=g\left(s_{t}\right), \forall t \in \mathcal{T} ; \\
& s_{1}=s_{T+1}=\bar{s} ;
\end{aligned}
$$




$$
\begin{aligned}
& s^{l} \leq s_{t} \leq s^{u}, \forall t \in \mathcal{T} \\
& -I^{d} \leq i_{t} \leq I^{c}, \forall t \in \mathcal{T} .
\end{aligned}
$$

Notice in this VIAM model, we consider the dependency of OCV on SOC by constraint (3c). We also replace the limits of charging/discharging power rates with the limits of charging/discharging current rates, this corresponds to constraint (3f). We note in VIAM model, in order to compare the solution quality, we assume that the SOC levels $s$ equal at the beginning and the end of studied time interval $\mathcal{T}$, i.e., $s_{1}=s_{T+1}$. A specific choice is $s_{1}=s_{T+1}=\bar{s}=0.5$. Under such an assumption, the profit comes only from the trading of electricity.

As shown in the survey papers [17]-[20] and the references therein, the OCVDF $g(s)$ is usually highly nonlinear and non-convex. The non-linearity and non-convexity in both the constraint functions and the objective function pose a tremendous computational challenge to even obtain a local optimal solution. To tackle such a challenge, we propose to approximate the OCVDF $g(s)$ by some relatively simple functions. For example, if we assume the OCVDF $g(s)$ is a constant, then the above model can be reduced to convex quadratic optimization which can be solved by many off-shelf optimization software such as [24]. In this paper, we will consider the scenario where the OCVDF $g(s)$ is approximated by a linear function and discuss how to solve the resulting bilinear optimization model in the following section.

\section{A Non-COnVEx Quadratic Programming Model AND REFORMULATION TECHNIQUES}

In this section, we consider a special case of VIAM model (3a) where the OCVDF $g(s)$ is an linear function. This leads to a non-convex quadratic programming model with linear and bi-linear constraints (BLCQP). We then introduce a novel nonlinear transformation to reformulate the original BLCQP model as another equivalent optimization problem with linear constraints and exponential objective function (LCEO).

\section{A. A Quadratic Programming Model Based on Linear Approx- imation of $O C V D F$}

In this subsection, we discuss how to solve model (3a) where the OCVDF is linear or approximated by an linear function. Let $g(s)=c_{0}+c_{1} s$ denote the linear approximate function. By applying the relation defined by constraint (3b) to the function $g(s)$, we obtain a new constraint as follows

$$
\begin{aligned}
g\left(s_{t+1}\right) & =g\left(s_{t}+\frac{\Delta}{E C} v_{t}^{o c} i_{t}\right) \\
& =g\left(s_{t}\right)+c_{1} \frac{\Delta}{E C} v_{t}^{o c} i_{t} \\
& =g\left(s_{t}\right)+c_{1} \frac{\Delta}{E C} g\left(s_{t}\right) i_{t} .
\end{aligned}
$$

Let $g^{l}=c_{0}+c_{1} s_{l}$ and $g^{u}=c_{0}+c_{1} s_{u}$ be the lower and upper bound for the linear OCVDF $g(s)$, then we can replace the box constraint (3e) on SOC by that of OCVDF $g(s)$. For notational convenience, let

$$
\tau=c_{1} \frac{\Delta}{E C}, \quad \bar{g}=g(\bar{s}), \quad g_{t}=g\left(s_{t}\right), \quad \forall t \in \mathcal{T} .
$$

Then we obtain the following quadratic programming model with linear and bi-linear constraints (BLCQP):

$$
\begin{aligned}
(B L C Q P) \min _{i_{t}, g_{t}} & \sum_{t \in \mathcal{T}} \lambda_{t}\left(g_{t} i_{t}+i_{t}^{2} R\right) \\
\text { s.t. } \quad & g_{t+1}=g_{t}+\tau g_{t} i_{t}, \forall t \in \mathcal{T} ; \\
& g_{1}=g_{T+1}=\bar{g} ; \\
& g^{l} \leq g_{t} \leq g^{u}, \forall t \in \mathcal{T} ; \\
& -I^{d} \leq i_{t} \leq I^{c}, \forall t \in \mathcal{T} .
\end{aligned}
$$

We have

\section{Theorem 1. Model (4) is equivalent to model (3).}

Proof. The sufficiency follows from our deduction of the BLCQP model and thus it remains to prove the necessity. Suppose that $g_{t}$ is a feasible solution to model (4a). Since $g(s)$ is a linear bijection, its inverse function $g^{-1}$ exists and is also linear. By applying $g^{-1}$ to both sides of constraint (4b), we obtain

$$
g^{-1}\left(g_{t+1}\right)=g^{-1}\left(g_{t}+\tau g_{t} i_{t}\right)=g^{-1}\left(g_{t}\right)+\frac{\tau}{c_{1}} g_{t} i_{t}
$$

which is equivalent to constraint (3b). Therefore, we obtain a solution $s_{t}=g^{-1}\left(g_{t}\right), \forall t \in \mathcal{T}$ to model (3a). This completes the proof of the theorem.

Note that in model (4a), there are some non-convex quadratic (or bilinear) terms in both objective function and constraint (4b). This implies that it is still nontrivial to solve model (4a) due to the presence of the non-convex quadratic functions.

\section{B. A Nonlinear Transformation}

In this subsection we introduce a nonlinear transformation to simplify the bilinear constraints in model (4a). For this, we note that in the constraint (4b), if we take the logarithm on both sides, we obtain:

$$
\log \left(g_{t+1}\right)=\log \left(g_{t}\right)+\log \left(1+\tau i_{t}\right)
$$

Let

$$
y_{t}=\log \left(g_{t}\right), z_{t}=\log \left(1+\tau i_{t}\right), \forall t \in \mathcal{T},
$$

we can rewrite the constraint (4b) as

$$
y_{t+1}=y_{t}+z_{t}, \forall t \in \mathcal{T} \text {. }
$$

Moreover, constraint (4c) can be rewritten as $y_{1}=y_{T+1}$ equivalently. Recall that $g_{t}=e^{y_{t}}$ and $i_{t}=\frac{e^{z}-1}{\tau}$, we reformulate the objective function by using constraint (4b), and new variables $y_{t}, z_{t}$,

$$
\begin{aligned}
\lambda_{t}\left(g_{t} i_{t}+i_{t}^{2} R\right) & =\lambda_{t}\left(\frac{g_{t+1}-g_{t}}{\tau}+i_{t}^{2} R\right) \\
& =\frac{\lambda_{t}}{\tau}\left(e^{y_{t+1}}-e^{y_{t}}\right)+\frac{\lambda_{t} R}{\tau^{2}}\left(e^{z_{t}}-1\right)^{2}
\end{aligned}
$$

Let

$$
\begin{array}{r}
\bar{y}=\log \left(g_{1}\right)=\log \left(g_{T+1}\right)=\log (\bar{g}) ; \\
y^{l}=\log \left(g^{l}\right), y^{u}=\log \left(g^{u}\right) ; \\
z^{l}=\log \left(1-\tau I^{d}\right), z^{u}=\log \left(1+\tau I^{c}\right) .
\end{array}
$$


Using the new variables $y_{t}$ and $z_{t}$ as defined in (5), we can recast model (4a) as the following optimization problem with linear constraints (LCEO):

$$
\begin{aligned}
(L C E O) \min _{y_{t}, z_{t}} & \sum_{t \in \mathcal{T}} \frac{\lambda_{t-1}-\lambda_{t}}{\tau} e^{y_{t}}+\frac{\lambda_{t} R}{\tau^{2}}\left(e^{z_{t}}-1\right)^{2} \\
\text { s.t. } \quad & y_{t+1}=y_{t}+z_{t}, \forall t \in \mathcal{T} \\
& y_{1}=\bar{y} \\
& y_{T+1}=\bar{y} \\
& y^{l} \leq y_{t} \leq y^{u}, \forall t \in \mathcal{T} \\
& z^{l} \leq z_{t} \leq z^{u}, \forall t \in \mathcal{T} .
\end{aligned}
$$

We remark that comparing with the original bilinear optimization model (4), model (6) has only linear constraints with an exponential objective function. Notice that, if

$$
\lambda_{t-1} \geq \lambda_{t}, \quad e^{z_{t}} \geq \frac{1}{2}, \quad \forall t \in \mathcal{T},
$$

then model (6) becomes a convex optimization problem. Particularly, because $e^{z_{t}} \geq e^{z_{l}}=1-\tau I^{d}$, the relation $e^{z_{t}} \geq \frac{1}{2}$ is usually satisfied in our problem setting due to the fact that $\tau I^{d}$ is much smaller than 1 . It also worths mentioning that when the prices remain invariant, i.e., $\lambda_{1}=\lambda_{2}=\cdots=\lambda_{T}$, then model (6) has a trivial optimal solution $\left(y_{1}=y_{2}=\cdots=\right.$ $\left.y_{T}, z_{1}=z_{2}=\cdots=z_{T}=0\right)$. This implies that a flat price dynamic will lead to less profit in the BESS.

\section{A Sequential Linear/QuAdratic Programming APPROACH}

In this section, we propose a sequential linear/quadratic programming approach (SLQP) for the reformulated LCEO model introduced in the previous section and establish its convergence.

To start, we mention that the solution $\left(y^{(0)}, z^{(0)}\right)=\left(y_{1}=\right.$ $\left.y_{2}=\cdots=y_{T+1}, z_{1}=z_{2}=\cdots=z_{T}=0\right)$ is feasible for the LCEO and it strictly satisfies the two box constraints.

Suppose at the current iterate $k$, a feasible solution $\left(y^{(k)}, z^{(k)}\right)$ is available. For every $t \in \mathcal{T}$, let

$$
\begin{aligned}
& f_{1}\left(y_{t}\right)=\frac{\lambda_{t-1}-\lambda_{t}}{\tau} e^{y_{t}}, \quad f_{2}\left(z_{t}\right)=\frac{\lambda_{t} R}{\tau^{2}}\left(e^{z_{t}}-1\right)^{2} ; \\
& f_{1}^{\prime}\left(y_{t}\right)=\frac{\lambda_{t-1}-\lambda_{t}}{\tau} e^{y_{t}}, \quad f_{2}^{\prime}\left(z_{t}\right)=\frac{\lambda_{t} R}{\tau^{2}}\left(2 e^{2 z_{t}}-2 e^{z_{t}}\right) ; \\
& f_{1}^{\prime \prime}\left(y_{t}\right)=\frac{\lambda_{t-1}-\lambda_{t}}{\tau} e^{y_{t}}, \quad f_{2}^{\prime \prime}\left(z_{t}\right)=\frac{\lambda_{t} R}{\tau^{2}}\left(4 e^{2 z_{t}}-2 e^{z_{t}}\right) .
\end{aligned}
$$

As pointed out in the previous subsection, the function $f_{2}\left(z_{t}\right)$ is convex w.r.t. $z_{t}$ as $e^{z_{t}} \geq \frac{1}{2}$ under the setting for the problem in this paper. Therefore, we can approximate $f_{2}\left(z_{t}\right)$ in a neighborhood of $z_{t}^{k}$ via its Taylor expansion as follows:

$$
\bar{f}_{2}\left(z_{t}^{k}+d_{z}^{t, k}\right)=f_{2}\left(z_{t}^{k}\right)+f_{2}^{\prime}\left(z_{t}^{k}\right) d_{z}^{t, k}+\frac{f_{2}^{\prime \prime}\left(z_{t}^{k}\right)}{2}\left(d_{z}^{t, k}\right)^{2} .
$$

Since the function $f_{1}\left(y_{t}\right)$ is only convex when $\lambda_{t-1} \geq \lambda_{t}$, and non-convex when $\lambda_{t-1}<\lambda_{t}$, we suggest to approximate $f_{1}($. in some neighborhood of $y_{t}^{(k)}$ via the following function

$\bar{f}_{1}\left(y_{t}^{k}+d_{y}^{k, t}\right)=\left\{\begin{array}{cc}f_{1}\left(y_{t}^{k}\right)+f_{1}^{\prime}\left(y_{t}^{k}\right) d_{y}^{k, t}+\frac{f_{1}^{\prime \prime}\left(y_{t}^{k}\right)}{2}\left(d_{y}^{k, t}\right)^{2} & \text { If } \lambda_{t-1} \geq \lambda_{t} ; \\ f_{1}\left(y_{t}^{k}\right)+f_{1}^{\prime}\left(y_{t}^{k}\right) d_{y}^{k, t} & \text { Otherwise. }\end{array}\right.$
To find a search direction $\left(d_{y}^{k}, d_{z}^{k}\right)$, we propose to solve the following convex quadratic optimization problem

$$
\begin{array}{ll}
\min _{d_{y}^{t, k}, d_{z}^{t, k}} \sum_{t \in \mathcal{T}} \bar{f}_{1}\left(y_{t}^{k}+d_{y}^{t, k}\right)+\bar{f}_{2}\left(z_{t}^{k}+d_{z}^{t, k}\right) \\
\text { s.t. } \quad d_{y}^{t+1, k}=d_{y}^{t, k}+d_{z}^{t, k}, \forall t \in \mathcal{T} ; \\
\quad d_{y}^{1, k}=0 ; \\
\quad d_{y}^{T+1, k}=0 ; \\
\quad y^{l} \leq y_{t}^{k}+d_{y}^{t, k} \leq y^{u}, \forall t \in \mathcal{T} ; \\
\quad z^{l} \leq z_{t}^{k}+d_{z}^{t, k} \leq z^{u}, \forall t \in \mathcal{T} .
\end{array}
$$

Theoretically, the above problem is polynomially solvable [25]. In our experiments, we use the optimization software CVX [24] and IPOPT [26] to solve the above problem. Once a search direction is identified, we need to find a step size $\alpha$ satisfying the following relation to ensure sufficient descent:

$$
\begin{aligned}
& \sum_{t \in \mathcal{T}} f_{1}\left(y_{t}^{k}+\alpha d_{y}^{k, t}\right)+f_{2}\left(z_{t}^{k}+\alpha d_{z}^{t, k}\right) \\
& \quad \leq \sum_{t \in \mathcal{T}} f_{1}\left(y_{t}^{k}\right)+f_{2}\left(z_{t}^{k}\right)+\frac{\alpha}{2}\left(f_{1}^{\prime}\left(y_{t}^{k}\right) d_{y}^{t, k}+f_{2}^{\prime}\left(z_{t}^{k}\right) d_{z}^{t, k}\right)
\end{aligned}
$$

Because the derivatives of $\bar{f}_{i}$ and $f_{i}$ always coincide with each other, we do not distinguish $\bar{f}_{i}^{\prime}$ and $f_{i}^{\prime}$ in inequality (8) and in the remaining parts of this work. Particularly, we adapt the Armijo-Goldstein line search procedure [27] for nonlinear optimization to find a suitable step size $\alpha$ as shown in algorithm 1.

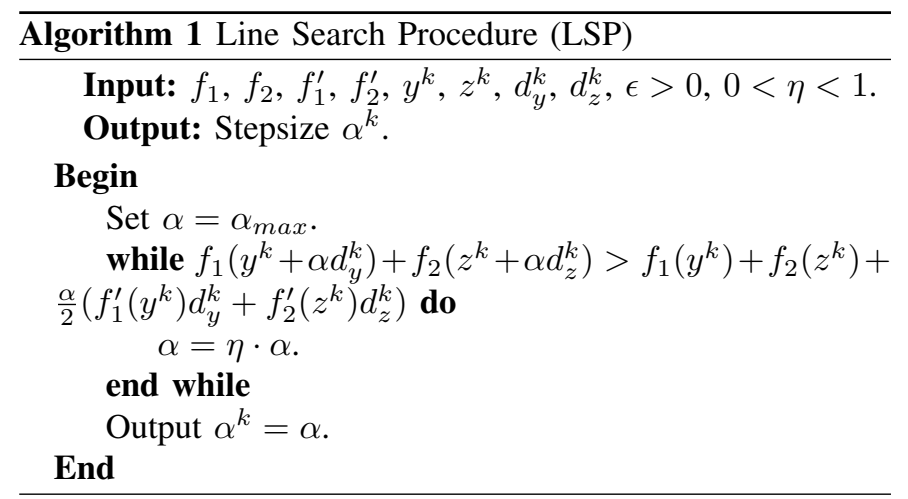

We point out that in the LSP algorithm 1, $\alpha$ is first set as the maximal allowable step size $\alpha_{\max }$. In our experiments, we choose $\alpha_{\max }=1$. We have

Theorem 2. The line search algorithm 1 can always find a step size $\alpha$ satisfying the inequality (8).

Proof. See Appendix A-A.

Now we are ready to describe the following sequential linear/quadratic programming (SLQP) approach for model (6) as shown in Algorithm 2. The SLQP is a modified variant of the classical sequential quadratic programming [27]. To explore the conditions under which the solution generated by the SLQP method is a local minimum, we need to rewrite the two box constraints (6e-6f) in the LCEO model as convex quadratic constraints. We can also eliminate the decision variables $\left\{z_{t}: t \in \mathcal{T}\right\}$ in the LCEO model via using the 
relation $z_{t}=y_{t+1}-y_{t}$. This leads to the optimization model (9a).

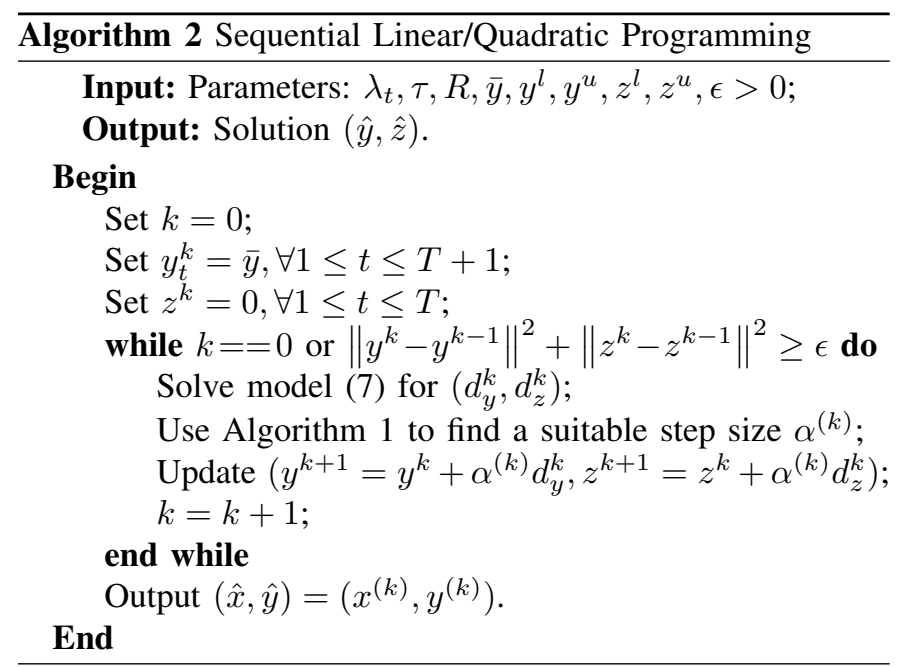

$$
\begin{array}{ll}
\min _{y_{t}} & \sum_{t \in \mathcal{T}} \frac{\lambda_{t-1}-\lambda_{t}}{\tau} e^{y_{t}}+\frac{\lambda_{t} R}{\tau^{2}}\left(e^{y_{t+1}-y_{t}}-1\right)^{2} \\
\text { s.t. } \quad & y_{1}=y_{T+1}=\bar{y} \\
& \left(y_{t}-y^{l}\right)\left(y_{t}-y^{u}\right) \leq 0, \forall t \in \mathcal{T} ; \\
& \left(y_{t+1}-y_{t}-z^{l}\right)\left(y_{t+1}-y_{t}-z^{u}\right) \leq 0, \forall t \in \mathcal{T} .
\end{array}
$$

Now let us consider the following Lagrangian function for the above problem:

$$
\begin{aligned}
\mathcal{L}\left(y_{t}, \gamma_{t}, \rho_{t}, \nu_{t}\right)= & \sum_{t \in \mathcal{T}} \frac{\lambda_{t-1}-\lambda_{t}}{\tau} e^{y_{t}}+\frac{\lambda_{t} R}{\tau^{2}}\left(e^{y_{t+1}-y_{t}}-1\right)^{2} \\
& +\gamma_{1}\left(y_{1}-\bar{y}\right)+\gamma_{T+1}\left(y_{T+1}-\bar{y}\right) \\
& +\sum_{t \in \mathcal{T}} \rho_{t}\left(y_{t}-y^{l}\right)\left(y_{t}-y^{u}\right) \\
& +\sum_{t \in \mathcal{T}} \nu_{t}\left(y_{t+1}-y_{t}-z^{l}\right)\left(y_{t+1}-y_{t}-z^{u}\right) .
\end{aligned}
$$

where $\left(\gamma_{t}, \rho_{t}, \nu_{t}\right)$ are the Lagrangian multipliers for constraints (9b), (9c) and (9d), respectively. We introduce the following regularity condition.

Condition 1 (Regularity). We say the solution $\left(y^{*}\right)$ satisfies the regularity condition, if

$$
H=\nabla_{y}^{2} L(y, \gamma, \rho, \nu) \succ 0
$$

where $H$ is a tri-diagonal matrix whose elements are defined as follows:

$$
\begin{aligned}
H_{t, t}= & \frac{2 R e^{-2\left(y_{t-1}+y_{t}\right)}}{\tau^{2}}\left[\lambda_{t-1} e^{3 y_{t}}\left(2 e^{y_{t}}-e^{y_{t-1}}\right)\right. \\
& \left.+\lambda_{t} e^{2 y_{t-1}+y_{t+1}}\left(2 e^{y_{t+1}}-e^{y_{t}}\right)\right] \\
& +2 \nu_{t-1}+2 \nu_{t}+2 \rho_{t}+\frac{\left(\lambda_{t-1}-\lambda_{t}\right) e^{y_{t}}}{\tau} \\
H_{t, t+1}= & H_{t+1, t}=\frac{2 \lambda_{t} R e^{y_{t+1}-2 y_{t}}\left(e^{y_{t}}-2 e^{y_{t+1}}\right)}{\tau^{2}}-2 \nu_{t}
\end{aligned}
$$

We have the following result.
Theorem 3. The SLQP algorithm 2 will generate a sequence $\left\{\left(y_{t}^{k}, z_{t}^{k}\right)\right\}$ converging to a stationary point $\left(y^{*}, z^{*}\right)$ of the LCEO problem (6). Moreover, if $\left(y^{*}, z^{*}\right)$ satisfies the regularity condition 1 , then $\left(y^{*}, z^{*}\right)$ is a local minimum of LCEO model (6).

Proof. See Appendix A-B

\section{EXPERIMENTAL RESULTS}

In this section, we first describe the data set used in our experiments, then run the simulation with 5-minute resolution to validate the efficacy of our new model and algorithms under different scenarios. Finally, we report all the simulation results. All numerical experiments are conducted on a laptop with 2.2 $\mathrm{GHz}$ six-core CPU and $16 \mathrm{~GB}$ memory. We use the 5-minute electricity price in year 2020 from MISO real-time market [28]. We use the battery data from Berrueta's paper [22]. Let

$$
x_{\alpha, a}=0.083+0.917 \cdot \mathrm{SOC} ; \quad x_{\alpha, c}=1-0.7 \cdot \mathrm{SOC} .
$$

The OCVDF used in [22] is defined as follows:

$$
\begin{aligned}
v_{e q}= & U_{c}^{0}-U_{a}^{0}+\frac{R \cdot T}{F} \cdot \ln \left(\frac{\left(1-x_{\alpha, c}\right) \cdot x_{\alpha, a}}{x_{\alpha, c} \cdot\left(1-x_{\alpha, a}\right)}\right) \\
& +\sum_{k=0}^{N} A_{k} \cdot\left[\left(2 \cdot x_{\alpha, c}-1\right)^{k+1}-\frac{2 \cdot x_{\alpha, c} \cdot k \cdot\left(1-x_{\alpha, c}\right)}{\left(2 \cdot x_{\alpha, c}-1\right)^{1-k}}\right] \\
& -\sum_{k=0}^{N} A_{k} \cdot\left[\left(2 \cdot x_{\alpha, a}-1\right)^{k+1}-\frac{2 \cdot x_{\alpha, a} \cdot k \cdot\left(1-x_{\alpha, a}\right)}{\left(2 \cdot x_{\alpha, a}-1\right)^{1-k}}\right] .
\end{aligned}
$$

For specific parameter values, we refer to the paper [22].

\section{A. VIAM VS LECO Simulation Results}

In this subsection, we compare the performance of the LCEO model, the VIAM model with a linear OCVDF (VIAML) and the VIAM model with a nonlinear OCVDF (VIAM$\mathrm{NL}$ ). To solve these models in reasonably large scale, we develop models and algorithms for the VIAM and LCEO by using Julia [29] with IPOPT [26] as the solver for the subproblems in the SLQP approach. In our experiments, the scale of the problem, measured by cardinality of $\mathcal{T}$, is determined by the number of days and price data resolution. For example, consider a scenario where the time span is 7 days and the price data resolution is 5-minutes, we have $T=7 * 24 * 12=2016$. To assess the effect from the fluctuation in electricity price on the profit, we solve the daily profits through year 2020, and calculate the mean and the standard deviation of the daily profits from each model. Table I compares the performance of three models (i.e., VIAM-L, VIAM-NL, and LCEO) in terms of the profit based on the control strategy derived from the obtained solution to the corresponding optimization model, where the average profits and the standard deviations are computed for a normalized battery with the capacity of $1 \mathrm{MW}$ per day.

From Table I we can observe that on average, the same amount of profit can be made based on the optimal solutions of both the LCEO and the VIAM-L models, while a slightly higher profit can be made based on the optimal solution to the VIAM-NL model. This shows that the usage of the linear 
TABLE I: Solutions from VIAM and LECO Models

\begin{tabular}{lllll}
\hline Days & T & Model & AVG Profit & SD of Profit \\
\hline \multirow{3}{*}{1} & \multirow{2}{*}{288} & VIAM-L & 59.2439 & 66.6185 \\
& & VIAM-NL & 59.3645 & 66.8112 \\
& & LCEO & 59.2439 & 66.6186 \\
\hline \multirow{3}{*}{7} & \multirow{2}{*}{2016} & VIAM-L & 59.7943 & 33.5843 \\
& & VIAM-NL & 59.9172 & 33.6756 \\
& & LCEO & 59.7943 & 33.5844 \\
\hline \multirow{3}{*}{30} & \multirow{3}{*}{8640} & VIAM-L & 60.2586 & 26.3356 \\
& & VIAM-NL & 60.3769 & 26.3337 \\
& & LCEO & 60.2532 & 26.2712 \\
\multirow{3}{*}{90} & \multirow{2}{*}{25920} & VIAM-L & 60.2586 & 13.4307 \\
& & VIAM-NL & 60.3823 & 13.4425 \\
& & LCEO & 60.2532 & 13.426 \\
\hline \multirow{3}{*}{180} & \multirow{3}{*}{51840} & VIAM-L & 60.2614 & 13.5676 \\
& & VIAM-NL & 60.3881 & 13.565 \\
& & LCEO & 60.2532 & 13.5666 \\
\hline \multirow{2}{*}{366} & \multirow{3}{*}{105408} & VIAM-L & 59.5409 & NA \\
& & VIAM-NL & 59.6659 & NA \\
& & LCEO & 59.5409 & NA \\
\hline \hline
\end{tabular}

approximation to the nonlinear OCVDF caused very few loss in profit.

Figure 3 shows the average CPU time used to solve each model versus the sizes of the solved instances in Julia. As we can see from Figure 3, the VIAM-NL model always requires the longest CPU time to solve, which indicates that the linear approximation to the nonlinear OCVDF does help to reduce substantially the difficulty of the underlying optimization model. We also observe that in Figure 3, while the CPU time used to solve each model increases as the problem size increases, and the proposed SLQP approach for the LCEO enjoys the slowest growth rate of the CPU time. Particularly, for the largest instance with over 300,000 decision variables, the CPU time used by the SLQP for the LCEO is less than $\frac{1}{3}$ of the CPU time used by IPOPT to solve the VIAM-L. This indicates that the SLQP is more efficient for large scale instances.

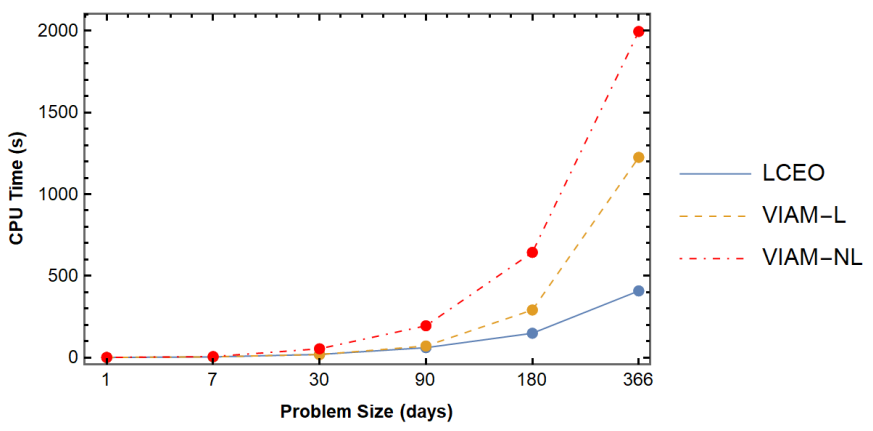

Fig. 3: Average CPU Time for VIAM and LECO Models

\section{B. Impact of Price Dynamics on LECO Solutions}

In this subsection, we discuss the impact of price dynamics on the LCEO model solutions and profits. Figure 4 shows the daily profits of a normalized battery under MISO and the daily price dynamic in 2020. As shown in Figure 4, there is a high volatility in the daily profits through the year. To assess the impact from the price dynamics on the daily profit, we select three days with the highest profits and three days with the lowest profits and plot the daily price dynamics for these 6 days in Figure 5 and 6. As shown in Figure 5 and 6, a lower price volatility usually leads to a lower profit. As pointed out in Section III, such a conclusion can also be drawn from the LCEO model (6) directly.

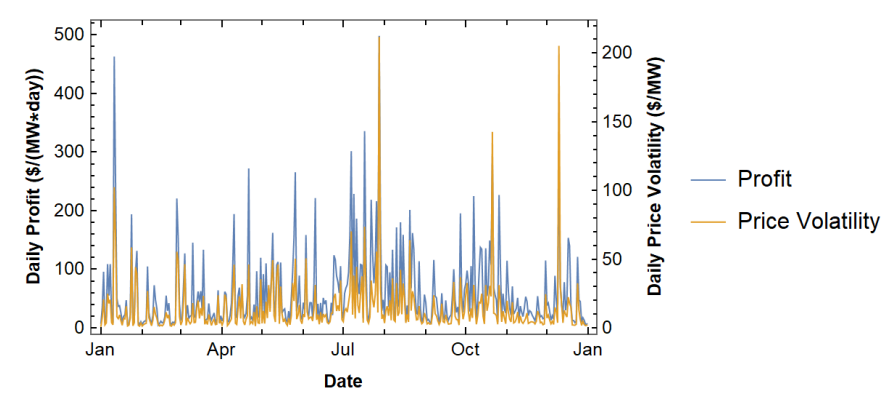

Fig. 4: Daily Arbitrage Profits in 2020

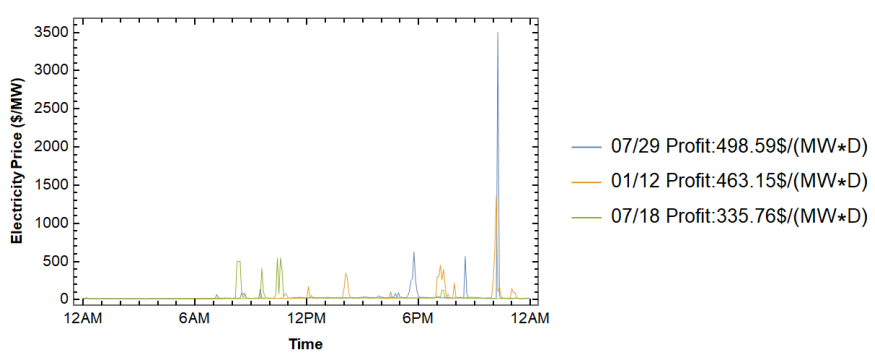

Fig. 5: Daily Price dynamics in Most Profitable Time

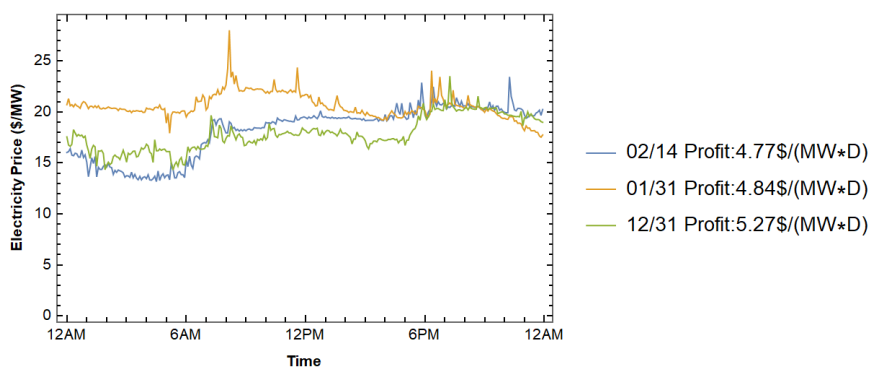

Fig. 6: Daily Price Dynamics in Least Profitable Time

Figure 7 shows the optimal BESS control strategy and MISO electricity price on July 10, 2021. This strategy follows a simple principal, i.e., to make more profits, the BESS should discharge the battery at high prices and charge the battery at low prices.

\section{CONCLUSION}

In this paper, we proposed a new BESS optimization model which naturally addresses the simultaneous charging/discharging problem by using a single current decision variable. We also proposed to use some linear function to approximate the highly nonlinear OCVDF to simplify the underlying BESS optimization model, which further leads to the new BLCQP model. To deal with the bilinear constraints in the BLCQP model, we introduced a novel reformulation technique to recast it as another equivalent LCEO with only 


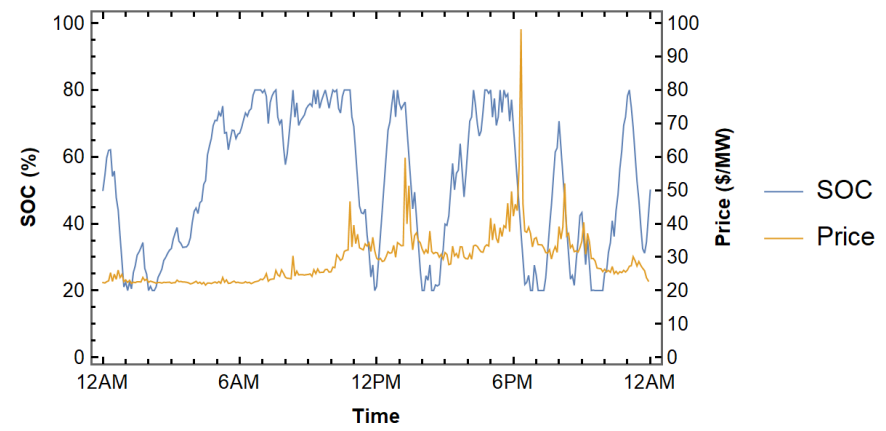

Fig. 7: SOC and Electricity Price on July 10, 2021

linear constraints. A new SLQP approach is proposed for the LCEO model and its convergence is established. Our preliminary experiments illustrate that the optimal solution from the new model provides a very good approximation to the optimal solution of the BESS optimization model with the original nonlinear OCVDF, and the proposed SLQP algorithm is competitive with the state-of-the-art optimization software such as IPOPT.

\section{APPENDIX A}

PROOFS OF THE THEOREMS

\section{A. Proof of Theorem 2}

Proof. Because $\left(d_{y}^{k, t}, d_{z}^{k, t}\right)$ is a descent direction, $\sum_{t \in \mathcal{T}} f_{1}^{\prime}\left(y_{t}^{k}\right) d_{y}^{t, k}+f_{2}^{\prime}\left(z_{t}^{k}\right) d_{z}^{t, k} \leq 0$. By Taylor's approximation, for sufficiently small $\alpha>0$, we have

$$
\begin{aligned}
& \sum_{t \in \mathcal{T}} f_{1}\left(y_{t}^{k}+\alpha d_{y}^{k, t}\right)+f_{2}\left(z_{t}^{k}+\alpha d_{z}^{t, k}\right) \\
= & \sum_{t \in \mathcal{T}} f_{1}\left(y_{t}^{k}\right)+f_{2}\left(z_{t}^{k}\right)+\sum_{t \in \mathcal{T}} \alpha\left(f_{1}^{\prime}\left(y_{t}^{k}\right) d_{y}^{t, k}+f_{2}^{\prime}\left(z_{t}^{k}\right) d_{z}^{t, k}\right) \\
& +o\left(\alpha \sum_{t \in \mathcal{T}} f_{1}^{\prime}\left(y_{t}^{k}\right) d_{y}^{t, k}+f_{2}^{\prime}\left(z_{t}^{k}\right) d_{z}^{t, k}\right) \\
= & \sum_{t \in \mathcal{T}} f_{1}\left(y_{t}^{k}\right)+f_{2}\left(z_{t}^{k}\right)+\sum_{t \in \mathcal{T}} \frac{\alpha}{2}\left(f_{1}^{\prime}\left(y_{t}^{k}\right) d_{y}^{t, k}+f_{2}^{\prime}\left(z_{t}^{k}\right) d_{z}^{t, k}\right) \\
& +\sum_{t \in \mathcal{T}} \frac{\alpha}{2}\left(f_{1}^{\prime}\left(y_{t}^{k}\right) d_{y}^{t, k}+f_{2}^{\prime}\left(z_{t}^{k}\right) d_{z}^{t, k}\right) \\
& +o\left(\alpha \sum_{t \in \mathcal{T}} f_{1}^{\prime}\left(y_{t}^{k}\right) d_{y}^{t, k}+f_{2}^{\prime}\left(z_{t}^{k}\right) d_{z}^{t, k}\right) \\
\leq & \sum_{t \in \mathcal{T}} f_{1}\left(y_{t}^{k}\right)+f_{2}\left(z_{t}^{k}\right)+\frac{\alpha}{2}\left(f_{1}^{\prime}\left(y_{t}^{k}\right) d_{y}^{t, k}+f_{2}^{\prime}\left(z_{t}^{k}\right) d_{z}^{t, k}\right) .
\end{aligned}
$$

This completes the proof.

\section{B. Proof of Theorem 3}

Proof. To start, we observe that the line search procedure 1 can always find an $\alpha$ satisfying the relation (8). Moreover, the termination of SLQP algorithm 2 is guaranteed, since the objective function value is monotonically decreasing and have finite lower bound (noting the box constraints for $y_{t}$ and $z_{t}$ ). Next, we prove the SLQP algorithm converges to a local minimum point $\left(y^{*}, z^{*}\right)$ of model (6). We first prove the SLQP algorithm converges to a stationary point $\left(y^{*}, z^{*}\right)$ of model (6). For notational simplicity, we convert constraints (6e-6f) to equivalent convex constraints $\left(y_{t}-y^{l}\right)\left(y_{t}-y^{u}\right) \leq 0$ and $\left(z_{t}-z^{l}\right)\left(z_{t}-z^{u}\right) \leq 0$. We note the constraints (6b-6f) satisfy the linear independence constraint qualification (LICQ). Because $\left(y^{*}, z^{*}\right)$ is a stationary point, it satisfies the following KKT conditions.

$$
\begin{array}{r}
\frac{\lambda_{t-1}-\lambda_{t}}{\tau} e^{y_{t}}-\mu_{t-1}+\mu_{t}+\rho_{t}\left(2 y_{t}-y^{l}-y^{u}\right)=0, \forall t \in \mathcal{T} ; \\
\frac{2 \lambda_{t} R_{e q}}{\tau^{2}}\left(e^{z_{t}}-1\right) e^{z_{t}}+\mu_{t}+\nu_{t}\left(2 z_{t}-z^{l}-z^{u}\right)=0, \forall t \in \mathcal{T} ; \\
\rho_{t}\left(y_{t}-y^{l}\right)\left(y_{t}-y^{u}\right)=0, \quad \rho_{t} \geq 0, \forall t \in \mathcal{T} ; \\
\nu_{t}\left(z_{t}-z^{l}\right)\left(z_{t}-z^{u}\right)=0, \quad \nu_{t} \geq 0, \forall t \in \mathcal{T} ; \\
\text { Constraints }(6 b-6 f)
\end{array}
$$

The SLQP algorithm 2 generates $(\hat{y}, \hat{z})$, which minimizes the quadratic objective function $\sum_{t \in \mathcal{T}} \bar{f}_{1}\left(y_{t}\right)+\bar{f}_{2}\left(z_{t}\right)$, subject to the constraints (6b-6f). Therefore, $(\hat{y}, \hat{z})$ satisfies the following KKT conditions.

$$
\begin{array}{r}
\bar{f}_{1}{ }^{\prime}\left(y_{t}\right)-\mu_{t-1}+\mu_{t}+\rho_{t}\left(2 y_{t}-y^{l}-y^{u}\right)=0, \forall t \in \mathcal{T} ; \\
\bar{f}_{2}{ }^{\prime}\left(z_{t}\right)+\mu_{t}+\nu_{t}\left(2 z_{t}-z^{l}-z^{u}\right)=0, \forall t \in \mathcal{T} ; \\
\rho_{t}\left(y_{t}-y^{l}\right)\left(y_{t}-y^{u}\right)=0, \quad \rho_{t} \geq 0, \forall t \in \mathcal{T} ; \\
\nu_{t}\left(z_{t}-z^{l}\right)\left(z_{t}-z^{u}\right)=0, \quad \nu_{t} \geq 0, \forall t \in \mathcal{T} ;
\end{array}
$$

Constraints $(6 b-6 f)$

Because

$$
\begin{aligned}
\bar{f}_{1}^{\prime}\left(\hat{y}_{t}\right) & = \begin{cases}f_{1}^{\prime}\left(\hat{y}_{t}\right)+f_{1}^{\prime \prime}\left(\hat{y}_{t}\right)\left(\hat{y_{t}}-\hat{y_{t}}\right) & \text { If } \lambda_{t-1} \geq \lambda_{t} \\
f_{1}^{\prime}\left(\hat{y_{t}}\right) & \text { Otherwise. }\end{cases} \\
& =f_{1}^{\prime}\left(\hat{y}_{t}\right) \\
\bar{f}_{2}^{\prime}\left(\hat{z}_{t}\right) & =f_{2}^{\prime}\left(\hat{z_{t}}\right)+f_{2}^{\prime \prime}\left(\hat{z}_{t}\right)\left(\hat{z_{t}}-\hat{z_{t}}\right)=f_{2}^{\prime}\left(\hat{z}_{t}\right)
\end{aligned}
$$

we observe the above KKT conditions coincide. Therefore, $(\hat{y}, \hat{z})$ is a stationary point for model (6).

Next, we prove that the stationary point is a local minimum. According to textbook [27], the local minimality holds true if the second order sufficient conditions are satisfied. We note that Condition 1 implies the second order sufficient conditions, this completes the proof.

\section{REFERENCES}

[1] R. Jiang, J. Wang, and Y. Guan, "Robust unit commitment with wind power and pumped storage hydro," IEEE Transactions on Power Systems, vol. 27, no. 2, pp. 800-810, 2011.

[2] D. Mohler and D. Sowder, "Energy storage and the need for flexibility on the grid," in Renewable Energy Integration. Elsevier, 2017, pp. 309-316.

[3] W. Cole, A. W. Frazier, and C. Augustine, "Cost projections for utility-scale battery storage: 2021 update," National Renewable Energy Lab.(NREL), Golden, CO (United States), Tech. Rep., 2021.

[4] M. R. Sheibani, G. R. Yousefi, M. A. Latify, and S. H. Dolatabadi, "Energy storage system expansion planning in power systems: a review," IET Renewable Power Generation, vol. 12, no. 11, pp. 1203-1221, 2018.

[5] X. Luo, J. Wang, M. Dooner, and J. Clarke, "Overview of current development in electrical energy storage technologies and the application potential in power system operation," Applied energy, vol. 137, pp. 511536, 2015. 
[6] M. Stecca, L. R. Elizondo, T. B. Soeiro, P. Bauer, and P. Palensky, "A comprehensive review of the integration of battery energy storage systems into distribution networks," IEEE Open Journal of the Industrial Electronics Society, vol. 1, pp. 46-65, 2020.

[7] Y. Wang, Y. Dvorkin, R. Fernandez-Blanco, B. Xu, T. Qiu, and D. S. Kirschen, "Look-ahead bidding strategy for energy storage," IEEE Transactions on Sustainable Energy, vol. 8, no. 3, pp. 1106-1117, 2017.

[8] S. Babaei, C. Zhao, and L. Fan, "A data-driven model of virtual power plants in day-ahead unit commitment," IEEE Transactions on Power Systems, vol. 34, no. 6, pp. 5125-5135, 2019.

[9] S. Hajiaghasi, A. Salemnia, and M. Hamzeh, "Hybrid energy storage system for microgrids applications: A review," Journal of Energy Storage, vol. 21, pp. 543-570, 2019.

[10] J. M. Arroyo, L. Baringo, A. Baringo, R. Bolaños, N. Alguacil, and N. G. Cobos, "On the use of a convex model for bulk storage in MIP-based power system operation and planning," IEEE Transactions on Power Systems, vol. 35, no. 6, pp. 4964-4967, 2020.

[11] Z. Li, Q. Guo, H. Sun, and J. Wang, "Sufficient conditions for exact relaxation of complementarity constraints for storage-concerned economic dispatch," IEEE Transactions on Power Systems, vol. 31, no. 2, pp. $1653-1654,2015$.

[12] S. Wang, G. Geng, and Q. Jiang, "Robust co-planning of energy storage and transmission line with mixed integer recourse," IEEE Transactions on Power Systems, vol. 34, no. 6, pp. 4728-4738, 2019.

[13] Y. Wen, C. Guo, H. Pandžić, and D. S. Kirschen, "Enhanced securityconstrained unit commitment with emerging utility-scale energy storage," IEEE Transactions on power Systems, vol. 31, no. 1, pp. 652-662, 2015.

[14] A. J. Gonzalez-Castellanos, D. Pozo, and A. Bischi, "Non-ideal linear operation model for li-ion batteries," IEEE Transactions on Power Systems, vol. 35, no. 1, pp. 672-682, 2019.

[15] H. Pandžić and V. Bobanac, "An accurate charging model of battery energy storage," IEEE Transactions on Power Systems, vol. 34, no. 2, pp. 1416-1426, 2018.

[16] A. Sakti, K. G. Gallagher, N. Sepulveda, C. Uckun, C. Vergara, F. J. de Sisternes, D. W. Dees, and A. Botterud, "Enhanced representations of lithium-ion batteries in power systems models and their effect on the valuation of energy arbitrage applications," Journal of Power Sources, vol. 342, pp. 279-291, 2017.

[17] P. Shrivastava, T. K. Soon, M. Y. I. B. Idris, and S. Mekhilef, "Overview of model-based online state-of-charge estimation using Kalman filter family for lithium-ion batteries," Renewable and Sustainable Energy Reviews, vol. 113, p. 109233, 2019.

[18] H. Hinz, "Comparison of lithium-ion battery models for simulating storage systems in distributed power generation," Inventions, vol. 4, no. 3, p. 41, 2019.

[19] D. M. Rosewater, D. A. Copp, T. A. Nguyen, R. H. Byrne, and S. Santoso, "Battery energy storage models for optimal control," IEEE Access, vol. 7, pp. 178 357-178 391, 2019.

[20] M. Shen and Q. Gao, "A review on battery management system from the modeling efforts to its multiapplication and integration," International Journal of Energy Research, vol. 43, no. 10, pp. 5042-5075, 2019.

[21] J. Tan and Y. Zhang, "Coordinated control strategy of a battery energy storage system to support a wind power plant providing multi-timescale frequency ancillary services," IEEE Transactions on Sustainable Energy, vol. 8, no. 3, pp. 1140-1153, 2017.

[22] A. Berrueta, A. Urtasun, A. Ursúa, and P. Sanchis, "A comprehensive model for lithium-ion batteries: From the physical principles to an electrical model," Energy, vol. 144, pp. 286-300, 2018.

[23] E. Zeidler, Nonlinear functional analysis and its applications: III: variational methods and optimization. Springer Science \& Business Media, 2013.

[24] M. Grant and S. Boyd, "CVX: Matlab software for disciplined convex programming, version 2.1," 2014

[25] D. G. Luenberger, Y. Ye et al., Linear and nonlinear programming. Springer, 1984, vol. 2.

[26] A. Wächter and L. T. Biegler, "On the implementation of an interiorpoint filter line-search algorithm for large-scale nonlinear programming," Mathematical programming, vol. 106, no. 1, pp. 25-57, 2006.

[27] J. Nocedal and S. Wright, Numerical optimization. Springer Science $\&$ Business Media, 2006

[28] MISO, "Midcontinent Independent System Operator (MISO)." [Online]. Available: https://www.misoenergy.org/

[29] J. Bezanson, A. Edelman, S. Karpinski, and V. B. Shah, "Julia: A fresh approach to numerical computing," SIAM Review, vol. 59, no. 1, pp. 65-98, 2017. 